\title{
Front Matter: Volume 10881
}

, "Front Matter: Volume 10881," Proc. SPIE 10881, Imaging, Manipulation, and Analysis of Biomolecules, Cells, and Tissues XVII, 1088101 (19 April 2019); doi: $10.1117 / 12.2523412$

SPIE. Event: SPIE BiOS, 2019, San Francisco, California, United States 


\section{Imaging, Manipulation, and Analysis of Biomolecules, Cells, and Tissues XVII}

Daniel L. Farkas

Attila Tárnok

James F. Leary

Editors

4-6 February 2019

San Francisco, California, United States

Sponsored and Published by

SPIE

Volume 10881 
The papers in this volume were part of the technical conference cited on the cover and title page. Papers were selected and subject to review by the editors and conference program committee. Some conference presentations may not be available for publication. Additional papers and presentation recordings may be available online in the SPIE Digital Library at SPIEDigitalLibrary.org.

The papers reflect the work and thoughts of the authors and are published herein as submitted. The publisher is not responsible for the validity of the information or for any outcomes resulting from reliance thereon.

Please use the following format to cite material from these proceedings:

Author(s), "Title of Paper," in Imaging, Manipulation, and Analysis of Biomolecules, Cells, and Tissues XVII, edited by Daniel L. Farkas, Attila Tárnok, James F. Leary, Proceedings of SPIE Vol. 10881 (SPIE, Bellingham, WA, 2019) Seven-digit Article CID Number.

ISSN: 1605-7422

ISSN: 2410-9045 (electronic)

ISBN: 9781510624047

ISBN: 9781510624054 (electronic)

Published by

SPIE

P.O. Box 10, Bellingham, Washington 98227-0010 USA

Telephone +1 3606763290 (Pacific Time) · Fax +1 3606471445

SPIE.org

Copyright (C) 2019, Society of Photo-Optical Instrumentation Engineers.

Copying of material in this book for internal or personal use, or for the internal or personal use of specific clients, beyond the fair use provisions granted by the U.S. Copyright Law is authorized by SPIE subject to payment of copying fees. The Transactional Reporting Service base fee for this volume is $\$ 18.00$ per article (or portion thereof), which should be paid directly to the Copyright Clearance Center (CCC), 222 Rosewood Drive, Danvers, MA 01923. Payment may also be made electronically through CCC Online at copyright.com. Other copying for republication, resale, advertising or promotion, or any form of systematic or multiple reproduction of any material in this book is prohibited except with permission in writing from the publisher. The CCC fee code is $1605-$ $7422 / 19 / \$ 18.00$.

Printed in the United States of America by Curran Associates, Inc., under license from SPIE.

Publication of record for individual papers is online in the SPIE Digital Library.

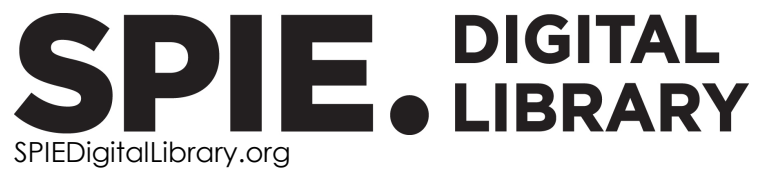

Paper Numbering: Proceedings of SPIE follow an e-First publication model. A unique citation identifier (CID) number is assigned to each article at the time of publication. Utilization of CIDs allows articles to be fully citable as soon as they are published online, and connects the same identifier to all online and print versions of the publication. SPIE uses a seven-digit CID article numbering system structured as follows:

- The first five digits correspond to the SPIE volume number.

- The last two digits indicate publication order within the volume using a Base 36 numbering system employing both numerals and letters. These two-number sets start with $00,01,02,03,04$, 05, 06, 07, 08, 09, 0A, OB ... 0Z, followed by 10-1Z, 20-2Z, etc. The CID Number appears on each page of the manuscript. 


\title{
Contents
}

\author{
vii Authors \\ ix Conference Committee
}

FUNCTIONAL IMAGING I

1088103 Improving quantitative fluorescence imaging with flat field illumination [10881-3]

FUNCTIONAL IMAGING ॥

1088106 Optical absorbance study of three phosphorylatable amino acids using confocal Raman Spectroscopy [10881-6]

1088109 High throughput analysis of plankton morphology and dynamic [10881-8]

MONITORING AND REGENERATIVE MEDICINE I

$10881 \mathrm{OB} \quad$ Design of portable microfluidic cytometry devices for rapid medical diagnostics in the field (Invited Paper) [10881-10]

10881 OC Dynamic observation and quantification of type $\mathrm{I} / \mathrm{ll}$ collagen in chondrogenesis of mesenchymal stem cells by second-order susceptibility microscopy (Invited Paper) [10881-11]

10881 OF Raman spectroscopy quality controls for GMP compliant manufacturing of tissue engineered cartilage [10881-14]

\section{MONITORING AND REGENERATIVE MEDICINE II}

$10881 \mathrm{OH} \quad$ Establishing the baseline for using plankton as biosensor [10881-16]

10881 ol Fluorescence molecular tomography based on L2,1-norm method for morphological reconstruction [10881-17]

10881 OK Cellular refractive index comparison of various prostate cancer and noncancerous cell lines via photonic-crystal biosensor [10881-19]

10881 OL Determination of extremely low concentration of sucrose in aqueous solution by Raman spectroscopy [10881-20] 
BIOMEDICAL IMAGING USING A DMD OR OTHER MIRROR ARRAY I: JOINT SESSION WITH 10881 AND 10932

$108810 \mathrm{~N} \quad$ A spherical mirror-based illumination system for fluorescence excitation-scanning hyperspectral imaging [10881-22]

1088100 Optimization of light transmission through an excitation-scan hyperspectral mirror array system [10881-23]

10881 OP Hyperspectral imaging and spatial frequency domain imaging: combined acquisition for full face skin analysis [10881-24]

\section{CYTOMICS I}

$108810 Q \quad$ Advancing methods for the analysis of glioblastoma cell motion using quantitative time lapse holographic imaging and cellular tomography (Invited Paper) [10881-25]

10881 OS External low frequency electric fields maximize the fluorescence enhancement through lightmetal-fluorophore interactions of target biomolecules [10881-28]

10881 OT The use of coated glass substrates for signal enhancement in multiphoton imaging [10881-29]

$108810 \mathrm{~V}$ Comparison of two- and three-dimensional nuclear to cytoplasm ratios in analyzing lung cancer cell lines [10881-27]

\section{CYTOMICS II}

$108810 X \quad$ Signal removal methods for highly multiplexed immunofluorescent staining using antibody conjugated oligonucleotides [10881-32]

$1088110 \quad$ Putting (single-cell) data into orbit [10881-35]

\section{SPECTRAL IMAGING}

$1088114 \quad$ Snapshot multi-spectral-line imaging for applications in dermatology and forensics [10881-39]

1088116 Raman imaging of a-synuclein aggregates in a rat model of Parkinson's disease [10881-41]

1088117 A multispectral Bayesian-based computational microscopy method for enhancing image quality [10881-42] 
1088119 Dark-field hyperspectral imaging of single plasmonic gold nanorods and their scattering characteristics in complex biological environments [10881-44]

10881 1A Single blood cell Raman spectroscopy reveals elevated haemoglobin content in poikilocytosis [10881-45]

10881 1B Optimizing channel selection for excitation-scanning hyperspectral imaging [10881-46]

10881 1C Multimodal optical detection and toxicity testing of microplastics in the environment [10881-47]

SPECTRAL IMAGING III

10881 1D Improvement of analyzing method for human skin color separation by independent component analysis [10881-48]

10881 1E Effect of different variables on indocyanine green (ICG) in image- guided treatments [10881-49]

$10881 \mathrm{lF} \quad$ Hyperspectral imaging microscopy for measurement of localized second messenger signals in single cells [10881-50]

\section{BIOINFORMATICS}

10881 1G Correlated simultaneous fluorescence and phosphorescence lifetime imaging reveals an association between intracellular oxygen tension and metabolic changes in living cells [10881-51]

$10881 \mathrm{lH} \quad$ High-resolution MR image by high precision signal analysis method for accurately analyze complex signals [10881-52]

$1088111 \quad$ Towards a Raman-based diagnostic approach for characterizing cytologically indeterminate thyroid nodules [10881-53]

$10881 \mathrm{lJ} \quad$ Bioluminescence tomography based on bilateral weight Laplace method for in vivo morphological imaging of glioma [10881-54]

$10881 \mathrm{~K} \quad$ Fast and robust reconstruction method for fluorescence molecular tomography based on deep neural network [10881-55]

$10881 \mathrm{lL} \quad$ Bone mineralization and collagen formation observed with principal component analysis of Raman scattering from healing calvarial defects [10881-56]

$108811 \mathrm{~N} \quad$ A novel method for scatterers type enumeration in polydisperse suspensions through fiber trapping and unsupervised scattering analysis [10881-58] 


\section{POSTER SESSION}

10881 1P Resolution-enhanced digital epiluminescence microscopy using deep computational optics [10881-60]

$10881 \mathrm{lQ}$ Increase of NMR/MIR signals under ultra-low B fields with hyperpolarized Xe using IW CW single-frequency Ti:Sapphire laser [10881-61]

10881 is Acoustic impedance measurement of radiotherapy-induced effect on the human tooth by 320 MHz scanning acoustic microscopy [10881-63]

10881 IT Multiphoton and harmonic generation imaging methods enable direct visualization of drug nanoparticle carriers in conjunction with vasculature in fibrotic prostate tumor mouse model [10881-64]

$10881 \mathrm{iU}$ Chemically etched plastic optical fiber probe for near-field scanning optical microscopy in liquids [10881-65]

10881 IW Towards laser-assisted microfluidic-cell transfection [10881-67] 


\section{Authors}

Numbers in the index correspond to the last two digits of the seven-digit citation identifier (CID) article numbering system used in Proceedings of SPIE. The first five digits reflect the volume number. Base 36 numbering is employed for the last two digits and indicates the order of articles within the volume. Numbers start with 00, 01, 02, 03, 04, 05, 06, 07, 08, 09, OA, OB...0Z, followed by 10-12, 20-2Z, etc.

Khaw, Ian, 03

Abraham, Thomas, $1 \mathrm{~T}$

Adair, James H., $1 \mathrm{~T}$

Adebiyi, Aminat, 09

Ahmed, Rafay, 1A, $1 \mathrm{~L}$

Algul, Emriye, is

An, Yu, Ol, 1J

Annamdevula, Naga, $1 \mathrm{~F}$

Barbero, Andrea, OF

Barroso, Álvaro, 1C

Batista, Ana, IW

Bianco, Simone, 09, $0 \mathrm{H}$

Bilen, Bukem, is

Biswas, Sujoy Kumar, 09, OH

Bozano, Luisa, 09

Brauchle, Eva, 16

Breunig, Hans Georg, IW

Brown, Cecelia, 09

Browning, Craig M., ON, 0O, $1 \mathrm{~F}$

Cadena, Melissa, OK

Campbell, Caroline A., 06

Campbell, Michael J., 11

Carvajal Berrio, Daniel, 16

Casadei, Nicolas, 16

Casey, Abigail H. M., 06

Ceylan, Cemile, is

Chan, James W., 11

Chang, Young-Hwan, OX

Chen, Huei-Wen, OV

Chen, Yang-Fang, OT, OV

Cherel, Marie, OP

Chiang, Shu-Jen, OV

Chin, Koei, OX

Clawson, Gary A., $1 \mathrm{~T}$

Clerc, Raphael, OP

Croop, Benjamin, 03

Crosta, Giovanni Franco, 10

Cunha, João P. S., $1 \mathrm{~N}$

Deal, Joshua, 1B, iF

Deglint, Jason L., 17

DeLuna, Frank, OK

Demirkan, Irem, is

Devireddy, Ram, 19

Dietler, Giovanni, $1 \mathrm{U}$

Dong, Chen-Yuan, OC, OT, OV

Dukenbayev, Kanat, $1 \mathrm{U}$

Edris, Wade, IT

Eng, Jennifer, OX

Fan, Yuxia, 11
Filiczak, Nina, $0 Q$

Fuchs, Ulrike, 03

Fuji, Kazuki, $1 \mathrm{H}$

Gao, Yuan, 0l, $1 \mathrm{~J}, 1 \mathrm{~K}$

Gartia, Manas Ranjan, 19

Gevaux, Lou, OP

Ghasempour, Askari, OL

Gibbs, Summer L., OX

Graß, Stefan, 1C

Gray, Joe, OX

Griswold, J. R., IF

Gunn Mayes, Samantha, ON, IF

Guo, Han-Wen, OT

Han, Kyu Young, 03

Han, Seung-Hee, $1 \mathrm{E}$

Hasan, Syed M., 19

Hasegawa, Masaya, $1 \mathrm{H}$

Hattori, Mineyuki, $1 Q$

Hébert, Mathieu, OP

Hirobayashi, Kanna, 1H

Hirobayashi, Shigeki, 1H

Hsueh, Chiu-Mei, OC

Huang, Chao, $1 \mathrm{~K}$

Huang, Eric C., 11

Huang, Hsu-Cheng, OV

Huang, Ya-De, $O C$

Jhaveri, Aditi, $O Q$

Jiang, Shixin, Ol, 1 J, 1K

Jin, Chao, 17

Jones, Jocelyn, OX

Jorge, Pedro A. S., IN

Juanda Ruha, Ahmad Naif Syaihan Bin, 1H

Kabiljagic, Dino, $1 \mathrm{P}$

Kalinina, Sviatlana, $1 \mathrm{G}$

Kemper, Björn, 1C

Ketelhut, Steffi, 1C

Kim, Ain, 1E

König, Aisada, IW

König, Karsten, IW

Kumagai, Hiroshi, 1Q

Kwon, Sunjong, OX

Lau, Condon, 1A, 1L

Lauberts, Kalvis, 14

Leary, James F., OB

Leavesley, Silas J., 0N, 00, 1B, 1F

Lee, Hsuan-Shu, OC

Lee, Pei-Jung, OV

Lee, Sheng-Lin, OC, OT

Lilge, Lothar, $1 G$ 
Lima, Ivan T., OS

Liu, Jie, Ol

Luther, Ed, OQ

Maini, Lucrezia, 09

Mandel, Arkady, $1 \mathrm{G}$

Martin, Ivan, OF

Matters, Gail L., $1 T$

Mayes, Samuel A., ON

McAlister, Kathleen, IF

McGovern, Christopher O., IT

McMahon, Nathan, OX

Mehta, Nishir, 19

Mendes, Livia, OQ

Meng, Hui, 1 J, 1K

Milane, Lara, $\mathrm{OQ}$

Möhl, Anna, 03

Nawarathna, Dharmakeerthi, OS

Ndi, Francis, OL

Nederlof, Michel, OX

Noguchi, Kyo, $1 \mathrm{H}$

Ogawa, Emiyu, 1Q

Oshina, Ilze, 14

Paiva, Joana S., IN

Pan, Xinhua, OL

Parker, Marina, 0N, 00, IF

Parlak, Melita, is

Pastore, Vito Paolo, 09, $0 \mathrm{H}$

Potapovs, Peteris, 14

Power, Laura, OF

Price, Alain, $\mathrm{OL}$

Rahimi, Nassim, OL

Reiber, Jens, $1 \mathrm{C}$

Ribeiro, Rita S. R., IN

Rich, Thomas C., ON, 0O, 1B, 1F

Riess, Olaf, 16

Rosa, Carla C., iN

Rueck, Angelika, $1 \mathrm{G}$

Sahu, Sushant, 19

Salker, Madhuri S., 16

Sampaio, Paula, $1 \mathrm{~N}$

Schenke-Layland, Katja, 16

Schnekenburger, Jürgen, 1C

Sekatskii, Sergey K., $1 \mathrm{U}$

Séroul, Pierre, OP

Sevgi, Fide, 16

Shaik, Shahensha, 19

Singh, Yogesh, 16

Smirnov, Anton, $1 \mathrm{U}$

Soares de Oliveira, Marcos A. S., 11

Spigulis, Janis, 14

Sun, Lu-Zhe, OK

Takao, Keizo, $1 \mathrm{H}$

Takeda, Shun, $1 Q$

Tanaka, Satomi, ID

Tang, Xiaomeng, $1 T$

Tang, Jialei, 03

Thibault, Guillaume, 0X

Tian, Jie, Ol, $1 \mathrm{~J}, 1 \mathrm{~K}$

Torchilin, Vladimir, $0 Q$

Tosi, Daniele, $1 \mathrm{U}$
Trémeau, Alain, $\mathrm{OP}$

Triplett, Gregory E., 06

Tsui, Suet Man, 1 A

Tsumura, Norimichi, 1D

Unlu, Mehmet Burcin, is

Velmanickam, Logeeshan, OS

Wang, Bingzhi, OK

Wang, Kung, Ol, 1J, $1 \mathrm{~K}$

Wen, Shu-Han, OV

Wendt, David, OF

Wixmerten, Anke, OF

Wong, Alexander, 17, 1P

Yang, Chang-Yong, $1 \mathrm{E}$

Yaprak, Gokhan, $1 \mathrm{~S}$

Ye, Jing Yong, OK

Zimmerman, Thomas, 09, $\mathrm{OH}$

viii

Proc. of SPIE Vol. 10881 1088101-8 


\section{Conference Committee}

Symposium Chairs

James G. Fujimoto, Massachusetts Institute of Technology (United States)

R. Rox Anderson, Wellman Center for Photomedicine, Massachusetts General Hospital (United States) and Harvard Medical School (United States)

Symposium Co-chairs

Jennifer K. Barton, The University of Arizona (United States)

Wolfgang Drexler, Medical University of Vienna (Austria)

Program Track Chairs

Ammasi Periasamy, University of Virginia (United States)

Daniel L. Farkas, University of Southern California (United States) and SMI (United States)

Conference Chairs

Daniel L. Farkas, University of Southern California (United States) and SMI (United States)

Attila Tárnok, Universität Leipzig (Germany)

Conference Co-chair

James F. Leary, Purdue University (United States)

Conference Program Committee

Vadim Backman, Northwestern University (United States)

Christopher H. Contag, Michigan State University (United States)

Paul M. W. French, Imperial College London (United Kingdom)

Yuval Garini, Bar-llan University (Israel)

Jae Youn Hwang, DGIST (Korea, Republic of)

Robert C. Leif, Newport Instruments (United States)

Charles P. Lin, Wellman Center for Photomedicine (United States)

Sacha Loiseau, Mauna Kea Technologies (France)

Dan V. Nicolau, McGill University (Canada)

Ramesh Raghavachari, U.S. Food and Drug Administration

(United States) 
Sebastian Wachsmann-Hogiu, University of California, Davis (United States)

Warren S. Warren, Duke University (United States)

\section{Session Chairs}

1 Functional Imaging I

Daniel L. Farkas, University of Southern California (United States) and SMI (United States)

2 Functional Imaging II

Daniel L. Farkas, University of Southern California (United States) and SMI (United States)

3 Monitoring and Regenerative Medicine I

Attila Tárnok, Universität Leipzig (Germany)

$4 \quad$ Monitoring and Regenerative Medicine II

Fartash Vasefi, eTreat Medical Diagnostics Inc. (Canada)

5 Biomedical Imaging using a DMD or Other Mirror Array I: Joint Session with 10881 and 10932

Michael R. Douglass, Texas Instruments Inc. (United States)

6 Biomedical Imaging using a DMD or Other Mirror Array II: Joint Session with 10881 and 10932

Roland Höfling, ViALUX GmbH (Germany)

7 Cytomics I

Attila Tárnok, Universität Leipzig (Germany)

8 Cytomics II

Attila Tárnok, Universität Leipzig (Germany)

9 Spectral Imaging I

Daniel L. Farkas, University of Southern California (United States) and SMI (United States)

James F. Leary, Purdue University (United States)

10 Spectral Imaging II

Daniel L. Farkas, University of Southern California (United States) and SMI (United States)

James F. Leary, Purdue University (United States) 
11 Spectral Imaging III

Daniel L. Farkas, University of Southern California (United States) and SMI (United States)

James F. Leary, Purdue University (United States)

12 Bioinformatics

James F. Leary, Purdue University (United States)

Proc. of SPIE Vol. 10881 1088101-11

Downloaded From: https://www.spiedigitallibrary.org/conference-proceedings-of-spie on $25 \mathrm{Apr} 2023$ Terms of Use: https://www.spiedigitallibrary.org/terms-of-use 
Proc. of SPIE Vol. 10881 1088101-12 Downloaded From: https://www.spiedigitallibrary.org/conference-proceedings-of-spie on 25 Apr 2023
Terms of Use: https://www.spiedigitallibrary.org/terms-of-use 